\title{
Podiumsdiskussion: Wechselwirkung zwischen nationalem und internationalem Arbeits- und Sozialrecht und dem Recht der Europäischen Gemeinschaft
}

\author{
Moderation: Karl-Dieter MÖLLER
}

Möller: Herr Minister Müntefering, welche Auswirkungen sehen Sie nach den Beschlüssen des Gipfels von Brüssel (Juni 2007) auf die europäische Arbeits- und Sozialpolitik?

Müntefering: Ich habe die Hoffnung, dass wir einen vernünftigen Grundlagen- oder Reformvertrag bekommen, in dem der ganze Teil Sozialpolitik entsprechend den ursprünglichen Regelungen des nicht in Kraft getretenen Verfassungsvertrags aufgenommen wird. Das bedeutet, dass es mit der Sozialklausel künftig für dieses Feld eine größere Aufmerksamkeit gibt, dass aber auch weiterhin bestimmte Bereiche wie der Kündigungsschutz ausgenommen bleiben, wo die nationalstaatliche Regelung die einzig mögliche ist. Ich glaube, dass wir insgesamt mit diesem Reformvertrag eine Stärkung der sozialen Dimension in Europa bekommen können.

Möller: In diesem Zusammenhang zwei Fragen an Sie, Herr Skouris. Es soll ja in dem neuen Vertrag festgelegt werden, dass es ab 2014 statt 27 Kommissaren nur noch 14 geben soll. Können Sie sich das auch für den Gerichtshof vorstellen? Stimmt es Sie ferner nachdenklich, dass England ausgeschert ist und zwar mit dem Argument, dass man sich sein Arbeitsrecht nicht kaputt machen lassen will?

Skouris: Was den britischen Vorbehalt im Hinblick auf die Grundrechtecharta anbelangt, so würde ich es begrüßen, wenn die Grundrechtecharta in der gesamten EU verbindlich wird. Zwar habe ich meinem heutigen Vortrag bereits ganz kurz erwähnt, dass sich dadurch eigentlich nichts Wesentliches an unserer Arbeit am Gerichtshof ändern würde. Dennoch wäre es eine Hilfe, einen modernen Grundrechtskatalog verbindlich in Kraft zu wissen. Zu dem britischen Vorbehalt im Konkreten möchte ich lediglich bemerken, dass es das Ergebnis unserer Rechtsprechung zu Gemeinschaftsgrundrechten auch im Hinblick auf die Solange-Rechtsprechung des Bundesverfassungsgerichts - ist, dass diese Grundrechte zunächst die Institutionen der Gemeinschaft binden, wenn sie ihre Kompetenzen ausüben, aber auch die Mitgliedstaaten, weil von diesen der größte Teil des Gemeinschaftsrechts angewendet wird. Das ist seit jeher die Position unsere Rechtsprechung und jetzt werden wir sehen, wie wir auf diese neue Entwicklung reagieren, sobald wir darüber zu entscheiden haben. 
Möller: Frau Koskelo, heute Vormittag hat Herr Skouris den meisten Beifall erhalten, als er sagte, dass 50 Jahre Rechtsprechung des EuGH gezeigt hätten, dass es auch ohne Charta gehe. Wie sehen Sie das als Präsidentin des Obersten Finnischen Gerichts?

Koskelo: Wenn wir uns die Entwicklung der Europäischen Union anschauen, dann ist es eine Selbstverständlichkeit, dass die Gemeinschaft, die anfing als eine Europäische Wirtschaftsgemeinschaft und sich dann in anderen Bereichen immer weiter entwickelt hat, auch die Anforderungen der Grundrechte gewährleisten muss, denn sonst hätten wir Verfassungskrisen in Mitgliedstaaten, die gezwungen wären, europäisches Recht anzuwenden, das im Gegensatz stünde zu den Grundrechten ihrer jeweils eigenen Verfassung und der Menschenrechtskonvention. Deswegen ist es eine unabdingbare Selbstverständlichkeit, dass die Grundrechte Teil der Rechtsprechung des EuGH geworden sind. Wir haben jetzt einen Punkt erreicht, wo diese Grundrechte in einem Rechtsinstrument der Union kodifiziert wurden, was eine ganz natürliche Entwicklung im Rechtssystem darstellt. Daher bin ich etwas besorgt, über diesen britischen Vorbehalt. Denn man kann sich zwar vorzustellen, dass ein Mitgliedstaat von einem bestimmten Themenbereich der Union, den man gut abgrenzen kann, ausgenommen ist, aber bei Grundrechten geht es um Querschnittsaufgaben, um eine große Bandbreite und nicht um einen eng definierten Bereich. Ich kann zwar verstehen, dass die britische Lösung aus politischen Gründen notwendig war, um eine ständige Reformkrise der Gemeinschaft zu verhindern. Dennoch glaube ich, dass es aus juristischer Sicht eine sehr problematische Lösung ist, die in den nächsten Jahren noch zu einigen Kopfschmerzen führen könnte.

Möller: Frau Schmidt, wie bewerten Sie als Präsidentin des Bundesarbeitsgerichts aber auch als europäische Bürgerin das, was am Wochenende in Brüssel beschlossen wurde. Gibt es ferner durch die Rechtsprechung des EuGH eigentlich einen Wandel vom Rechts- zum Richterstaat? Ich frage das mit dem Hintergedanken, dass auch das BAG ständig dafür kritisiert wird, Arbeitsrecht zum Richterecht gemacht zu haben. Fühlen Sie sich da mit Herrn Skouris seelenverwandt?

Schmidt: Ich fühle mich in einigen Punkten meiner Tätigkeit mit Herrn Skouris seelenverwandt, denn wir haben zunächst die gleiche Ausgangslage, da auch das Arbeitsrecht über viele unterschiedliche und diffuse Rechtsquellen verfügt. Also Gesetz, Tarifvertrag, Betriebsvereinbarungen und das Richterrecht für die Bereiche, in denen der Gesetzgeber keine Kraft findet, Regelungen zu treffen, gleichwohl Konflikte an die Richter herangetragen werden. Das führt dazu, dass das Arbeitsrecht von einer gewissen Unsystematik gekennzeichnet ist und schon bin ich beim Gemeinschaftsrecht, das klar geschriebene Rechtsquellen wie Grundlagenverträge, Verordnungen und Richtlinien kennt, aber eben auch eine Fülle von Richterrecht. Die weitere - bedauerliche - Gemeinsamkeit zwischen den beiden Rechtsgebieten ist die durch die Fülle an Richterecht 
hervorgerufene Intransparenz beider Systeme. Dass wieder Transparenz zugunsten politischer Kompromisse geopfert werden musste befremdet mich an den Ergebnissen des Gipfels. Das kann man aus Sicht der Politiker verstehen, es geht jedoch zu Lasten der Akzeptanz, so dass der Bürger Europa immer weniger versteht, was man ihm auch nicht verübeln kann. Denn die Rechtsquellenlage ist unklar und die Informationen, die der Bürger erhält machen dieses Informationsdefizit auch nicht wett.

Im Übrigen ist das, was das Vereinigte Königreich mit dem Vorbehalt bzgl. der Grundrechtecharta erwirkt hat, ein positives Signal dafür, dass die Kooperation zwischen dem EuGH und den nationalen Gerichten funktioniert. Denn das Vereinigte Königreich hat sein Verhalten nicht damit begründet, dass es den EuGH für völlig unberechenbar hält, sondern dass man nicht möchte, dass die nationalen Gerichte Gemeinschaftsrecht anwenden. Das ist für mich schon ein Unterschied und das Positive daran ist, dass Regierungen verstanden haben, dass eine Kooperation zwischen dem EuGH und den nationalen Gerichten stattfindet, dass die nationalen Gerichte auch Verantwortung für Europa tragen, wie umgekehrt der EuGH Teil des nationalen Rechtszugs geworden ist.

Möller: Sie haben ein wichtiges Stichwort gegeben, nämlich den europäische Bürger. Ein Kritiker hat einmal geschrieben, es reiche nicht aus, jeden morgen zum Frühstück das Amtsblatt der Europäischen Gemeinschaft zu lesen, sondern erforderlich sei noch in regelmäßigen Abständen den Wahrsager zu konsultieren, um sich die neuesten Wendungen der EuGH-Rechtsprechung voraussagen zu lassen. Würden Sie diese Aussage teilen, Frau Wendeling-Schröder?

Wendeling-Schröder: Nein, ganz gewiss nicht. Ich kann mir auch nicht vorstellen, dass das Amtsblatt der Europäischen Gemeinschaft eine erquickliche und weiterführende Morgenlektüre wäre. Was dahinter steckt, ist eine gewisse Befürchtung der Intransparenz und das ist ein Vorwurf, der dem EuGH naturgemäß anhaften wird, denn er ist nicht aus einer nationalen Gerichtsstruktur herausgewachsen und kann nicht auf ein mehr oder weniger einheitliches Rechtssystem zurückblicken. Er arbeitet nur mit den Materialien, die spezifisch gemeinschaftsrechtlich sind. Nämlich mit dem dürren Vertrag, bei dem jede Änderung die schon beschriebenen Schwierigkeiten aufwirft und den Folgerungen, die daraus zu ziehen sind. Das macht es relativ schwierig, eine dogmatisch konsistente Rechtsprechung vom EuGH festzustellen.

Möller: Wie bringen Sie ihren Studenten Europa oder den Europäischen Gerichtshof näher?

Wendeling-Schröder: Ich besuche hin und wieder mit Studierenden den Gerichtshof. Natürlich nehmen wir an der Universität auch von dessem Entscheidungen Notiz, denn die Rechtsprechung des EuGH gehört ja mittlerweile zu praktisch jedem juristischen 
Fach. Das Problem für die Studierenden ist, dass die Herangehensweise anders sein muss. Man hat ja auch eine andere Art von Urteilen. Die sind sehr kurz, für unsere Vorstellungen recht apodiktisch und nicht so begründet, wie wir das von der deutschen Rechtsprechung gewohnt sind. Das macht es den Studierenden vergleichsweise schwer, sie zu verstehen. Auch weil die dogmatischen Instrument e, die beim EuGH verwendet werden, sich aus verschiedenen nationalen Rechtsordnungen entwickelt haben.

Möller: Kurze Frage an Herrn Skouris: Halten Sie ihre Urteile, die Urteile des Europäischen Gerichtshofs für schwer verständlich?

Skouris: Ich will nicht ausschließen, dass sie manchmal gewisse Schwierigkeiten aufwerfen können. Man muss dabei auch bedenken, dass der Gerichtshof unter ganz spezifischen Bedingungen zu seinen Entscheidungen kommt. Dabei darf nicht verkannt werden, dass wir nicht immer mit einem Verfassungsgericht verglichen werden sollten, auch wenn wir gewisse, den Verfassungsgerichten ähnliche Zuständigkeiten haben. Aber wir sind - und das möchte ich einmal klarstellen - weder ein Verfassungsgericht im Sinne eines Gerichtshofs für Menschenrechte, noch ein Verfassungsgericht im Sinne des deutschen Bundesverfassungsgerichts. Unsere Hauptaufgabe ist vielmehr, für die einheitliche Anwendung und Auslegung des Gemeinschaftsrechts zu sorgen, eine Aufgabe also, die wir eher mit den höchsten Bundesgerichten teilen. Wenn man Vergleiche zwischen uns und diesen obersten Bundesgerichten anstellt, wird man feststellen, dass die Unterschiede nicht ganz so groß sind. Verfassungsgerichtliche Urteile sind lang und sehr ausführlich begründet. Wir hingegen müssen bei jeder Randnummer unserer Urteile genau diskutieren, ob der jeweilige Gedanke für das Ergebnis des konkreten Einzelfalles wirklich wichtig ist, weil wir dieser Gedanken vielleicht in einem anderen Fall, als obiter dictum eine faktische Bindung bewirken könnte. Abschließend möchte ich noch hinzufügen, dass gerade solche Tagungen beweisen, dass unsere Urteile von vorne bis hinten so genau gelesen werden, dass wir zu recht ganz vorsichtig sind mit dem, was wir schreiben, weil eben manchmal dem Gerichtshof auch einiges unterstellt wird

Möller: Würden Sie dem zustimmen, dass das, was der EuGH macht, tief in die Politik der Mitgliedsländer hineinwirkt?

Skouris: Das kann ich nicht ausschließen. Sicherlich spielt unsere Rechtsprechung eine wichtige Rolle in den Mitgliedstaaten, aber das liegt eben am heutigen rechtstaatlichen System mit einer ausgebauten Gerichtsbarkeit.

Möller: Herr Müntefering, Sie haben die Forderung nach sozialer und wirtschaftlicher Ausgewogenheit bei der Urteilsfindung durch den EuGH erhoben. Was gibt es an den bisherigen Urteilen in diesbezüglich von Seiten der Politik zu kritisieren? 
Müntefering: Das muss ich jetzt ganz vorsichtig sein. Denn wie Herr Präsident Skouris heute schon betont hat, kann der EuGH nur auf der Basis dessen entscheiden, was wir Politiker als gesetzliche Grundlage vorgegeben haben. Ich nähere mich dem ganzen Thema nicht juristisch, sondern als Politiker, der den Menschen erklären und vermitteln muss, was da in Europa stattfindet und der genau weiß, dass Entscheidungen der Politik, aber auch der Rechtsprechung, von entscheidender Bedeutung für die Akzeptanz dieses Europas bei den Menschen sind. Die Frage an uns Politiker ist, was wir eigentlich tun müssen, damit dieses Europa gelingen kann, was dann erreicht ist, wenn die Bürgerinnen und Bürger das Gefühl haben, dass hier Gerechtigkeit vorherrscht und dass sie auf europäischer Ebene zu ihrem Recht kommen. Mir als Sozialpolitiker stellt sich in diesem Zusammengang die Frage nach der sozialen Dimension Europas, ob also in den Urteilen des EuGH zu den europäisierten Bereichen der Wirtschafts- und Finanzpolitik, soziale und arbeitsrechtliche Fragen hinreichend berücksichtigt werden können, obwohl diese Themen nach wie vor in die Kompetenz der Mitgliedstaaten fallen, so dass es zu ausgewogenen, für die Menschen nachvollziehbaren Urteilen kommen kann. Da bin ich schon besorgt, dass es hier Diskrepanzen geben könnte. Das ist kein Vorwurf, sondern einfach das Bemühen, die Übersicht zu behalten in einem komplexen Vorgang, den Politiker nicht immer begreifen und ganz sicher nicht die Menschen auf der Straße und in dem wir lernen müssen, das, was wir heute hier unter Fachleuten besprechen, auch den Menschen verständlich zu machen. Das ist ein Problem der Politik, aber auch der Juristen. Ich weiß, dass man einsteinsche Formeln nicht mit „müntischen“ Sätzen umschreiben kann. Es ist trotzdem unsere Aufgabe, den Menschen zu erklären, um was es da in Europa geht. Allerdings verwenden wir in der Politik, aber auch Richterinnen und Richter oftmals eine Sprache, die nicht darauf Rücksicht nimmt, ob sie von den Menschen verstanden wird. Daraus entstehen dann schnell Missverständnisse und Irritationen und auch der leichtfertige Umgang mit einem Gericht, das seine hohen Verdienste hat. Zusammengefasst bin ich stets darum besorgt, dass dieses Europa richtig vermittelt wird und dass die unterschiedlichen Zuständigkeiten, Wirtschaft und Finanzen auf europäischer Ebene, Arbeit und Soziales auf mitgliedstaatlicher Ebene, zu einer vernünftigen und in sich kohärenten Politik und Rechtsprechung führen.

Möller: Sind Sie der Meinung, dass der Europäische Gerichtshof bei seinen Entscheidungen verstärkt die damit verbundenen sozialen Folgen berücksichtigen muss?

Müntefering: Da komme ich wieder zu dem eingangs erwähnten Punkt. Der Präsident sagt zu Recht, der EuGH entscheide auf der Grundlage, die die Politik per Gesetz vorgibt. Die Frage müsste dann eher an uns Politiker gerichtet sein: Sind die Zusammenhänge zwischen dem, was Europa beschließt und dem, was auf nationaler Ebene geregelt wird, so unzureichend, dass dadurch Komplikationen in der Rechtsprechung entstehen? Das ist dann etwas, wo die Politik unzureichend bleibt. Ich will es lieber auf uns Politiker nehmen, als es den Richtern zuzuschieben und vielleicht können wir uns 
als Politiker gemeinsam darum bemühen, die Dinge soweit zusammenzubringen, dass das Europarecht befriedigend angewendet werden kann, denn ansonsten geht es letztlich immer zu Lasten der Akzeptanz Europas bei den Menschen.

Möller: Herr Skouris, wäre Ihnen das nicht auch sehr recht, wenn die Politik so handeln würde, vielleicht kämen Sie dann viel schneller an die Zahl 15 beim EuGH?

Skouris: Das weiß ich nicht, ob wir dann noch schneller werden, aber ich stimme Herrn Müntefering voll und ganz in dieser allgemeinen Beurteilung der Lage zu. Darüber hinaus meine ich, dass wir Richter beim EuGH besonders für Fälle empfindlich sind, die soziale Bezüge aufweisen. Obwohl für das Arbeits- und Sozialrecht primär die Mitgliedstaaten zuständig sind, da es hier keine Übertragung von wichtigen Zuständigkeiten auf die Europäische Union vollzogen wurde, gibt es aber dennoch viele Fälle, die soziale Bezüge aufweisen, weil es sich dort um Wanderarbeitnehmer handelt, oder um Menschen, die von ihren Freiheitsrechten Gebrauch machen. Wenn man als Gerichtshof versucht, auch die soziale Komponente zu berücksichtigen und auf diese Weise den Menschen zu helfen, dann wird man bisweilen kritisiert, weil man dafür nicht zuständig sei. In dieser Argumentation sehe ich einen gewissen Widerspruch: wenn Europa sozialer werden soll und der Gerichtshof mangels speziellerer Regelungen bereits bestehende Rechtsgrundlagen, wie die wichtigen VO 1408/71 und 1612/68 anwendet und dabei erweiternd auslegt, dann sollte man nicht kritisieren, dass der Gerichtshof zu weit gegangen sei, weil das den Mitgliedstaaten obliege. Schließlich sind es die Gerichte der Mitgliedstaaten, die sich an uns wenden, damit wir eben diese Erweiterung vornehmen.

Möller: Genau, das war sozusagen die Übergangsfrage zu Frau Schmidt und auch Frau Koskelo. Das BAG, BSG, aber auch das finnische oberste Gericht sind ja aus der Sicht von Herrn Skouris eine Art Katalysator. Bei Ihnen landen zunächst die Widersprüche, die Politiker durch ihre Gesetze gemacht haben. Wie sehen Sie diese Funktion als oberste Gerichte in den Mitgliedstaaten auch in Bezug auf den EuGH? Eine weitere Frage ist dann, ob man nicht den obersten Gerichten, dem BAG, dem BSG die Rechtsprechung des Gemeinschaftsrechts vielleicht einmal irgendwann übertragen sollte, so dass der EuGH nur noch in ganz wenigen streitigen Fällen entscheiden würde?

Schmidt: Das ist heute schon der Fall. Weil der EuGH schon seit über 50 Jahren judiziert, hat eine ganze Reihe von Normen des Gemeinschaftsrechts eine Ausprägung und Klarheit erhalten und ist damit in der Auslegung für alle nationalen Gerichte handhabbar. Man sieht das auch daran, dass bei den nationalen Gerichten die Methode der europarechtskonformen Auslegung immer mehr an Bedeutung gewinnt. Das heißt, dass hier die Ergebnisse der bisherigen Rechtsprechung des Gerichtshofes nutzbar gemacht werden. Insoweit ist ein erneutes Anrufen des EuGH nicht nötig. Wenn ich mir im Üb- 
rigen so die ein oder andere Frage ansehe, die dem Gerichtshof vorgelegt wird, dann bleibt mir nur zu sagen: Don't ask so silly questions!

Möller: Sollte man also das Vorabentscheidungsverfahren abschaffen?

Schmidt: Nein. Das ist sicherlich ein wichtiges Verfahren vor dem EuGH im Sinne umfassenden Rechtschutzes. Allerdings waren die spektakulären Fälle an Vorabentscheidungsverfahren, die in den letzten Jahren zur Kritik am EuGH geführt haben, solche, in denen entweder die Nationalstaaten dem Frustrationsverbot zuwider gehandelt haben oder in denen es nicht zu einer Umsetzung von Richtlinien gekommen ist. Da frage ich mich, wieso Unionsbürger über den Weg ihrer Klage und damit über die Vorabentscheidung versuchen müssen eine Klärung herbeizuführen, um die Nationalstaaten zu bewegen, die Verpflichtungen, die sie auf europäischer Ebene eingegangen sind, auch einzuhalten. Das wäre doch vielmehr eine Aufgabe der Kommission in Fällen, in denen Richtlinien nicht umgesetzt wurden oder in denen offenkundig gegen das Frustrationsverbot verstoßen wurde, nicht zu lange mit dem Vertragsverletzungsverfahren abzuwarten.

Möller: Frau Koskelo, wie sehen Sie das?

Koskelo: Herr Müntefering hat zu Recht betont, dass die Bürger etwas Bestimmtes von Europa erwarten. Die nationalen Gerichte sind dabei die Vehikel, über die die Bürger das von Europa bekommen, was sie erwarten können. Denn die Gerichte müssen das europäische Recht durchsetzen und da gibt es eine klare Aufgabenteilung zwischen dem EuGH und den nationalen Gerichten. Die Aufgabe des EuGH besteht dabei darin, dass die Menschen überall in Europa die gleichen Rechte haben und Ansprüche erfüllt bekommen. Deshalb ist diese Aufgabenteilung der Schlüssel für das Funktionieren des gesamten europäischen Rechts. In diesem Zusammenhang empfinde ich den Mechanismus und das System der Vorabentscheidung als genial. Damit wird für die Zusammenarbeit vom EuGH mit den nationalen Gerichten ein institutioneller Rahmen geschaffen, in dem die Rolle der nationalen Gerichte sehr wichtig ist, da der EuGH nur die Fragen beantworten kann, die ihm die nationalen Gerichte vorlegen. Diese haben dabei eine große Verantwortung, da sie einerseits kritisch auf die innerstaatlichen Rechtsvorschriften schauen müssen, um herauszufinden, wo Hinweise vom EuGH benötigt werden, damit die Rechte der Bürger dann entsprechend realisiert werden können. Andererseits müssen sie bei der Vorlage zum EuGH in einer sehr umsichtigen Art und Weise handeln, denn wir kennen ja alle die Kriterien für Vorabentscheidungen.

Möller: Frau Wendeling-Schröder, der ehemalige Bundespräsident und Präsident des Bundesverfassungsgerichts, Prof. Herzog, hat über die Rechtsprechung des EuGH einmal gesagt, dass diese eine Ursache für die sachwidrige Zentralisierung der Union sei. 
Das Gericht betreibe, so die Kritik, die größtmögliche Ausschöpfung der Gemeinschaftsbefugnis, also im Zweifel immer für die Zuständigkeit der Union. Was sagen Sie zu dieser Kritik?

Wendeling-Schröder: Sie ist zwar ausgesprochen pointiert, greift jedoch das Kernproblem auf, nämlich die Frage nach den genauen Zuständigkeiten der EU in Abgrenzung zu den Mitgliedsstaaten. Das spiegelt sich wieder in der Politik des EuGH, der sich als Motor der Einigung auf europäischer Ebene verstanden hat. Aber es gelingt natürlich einer Organisation schlecht, immerzu die eigenen Grenzen zu sehen, so dass der EuGH hin und wieder die Grenzen ausweiten möchte, was auf einen gewissen Gegendruck stößt. $\mathrm{Zu}$ lösen ist dieser Konflikt nur durch eine konsequente Rückbesinnung auf die Rechtsgrundlagen, die dem EuGH zur Verfügung stehen. Denn der EuGH kann nicht in der gleichen Weise rechtsfortbildend tätig werden, wie ein nationales Gericht, das auf verschiedene Rechtsfiguren aus dem jeweiligen nationalen Umfeld zurückgreifen kann. Dies ist dem EuGH nicht möglich. Das zwingt dazu, sich vergleichsweise streng an die mehr oder weniger geschriebenen Rechtsgrundlagen zu halten.

Zudem ist das System der Rechtssetzung auf europäischer Ebene ein relativ kompliziertes, aber auch eines, was einen gewissen Charme hat. Es schafft es nämlich einerseits zu regeln und andererseits Regelungsspielräume für die Politik zu lassen. So wird beispielsweise bei der Richtlinie ein Ziel vorgegeben, das jeder Mitgliedstaat so umsetzen kann, wie er das politisch verantworten möchte. Aufgrund dieser Gestaltungsmöglichkeiten der Mitgliedstaaten bei der Richtlinienumsetzung, gibt es meines Erachtens keine Spielräume für Entscheidungen des EuGH, die den Mitgliedstaaten die konkrete Ausgestaltung der Richtlinienumsetzung vorgeben, solange die Umsetzungsfrist einer Richtlinie noch läuft.

Zusammenfassend lässt sich feststellten, dass der EuGH immens wichtig für Europa ist und dass man ihm nicht allzu vorschnell vorwerfen sollte, er habe die Grenzen seiner Kompetenz überschritten. Aber diese Grenzen sind dann deutlich aufzuzeigen, wenn sie erreicht sind, weil sonst das Tandem zwischen den Nationalgerichten und dem EuGH nicht mehr funktioniert.

Möller: Herr Müntefering, müssen Politiker nicht manchmal genauer hinschauen, was sie beschließen? Ich erinnere mich an ein Aha-Erlebnis beim Bundesverfassungsgericht, als es um den Europäischen Haftbefehl ging. Da zeigten sich Politiker aller Parteien, sichtlich überrascht über die unerwartet weitreichenden Folgen ihres Gesetzesbeschlusses. Sollten sich die Politiker nicht manchmal genauer überlegen, welche Kompetenzen sie an Europa abgeben und welche folgen das haben könnte, ehe sie den EuGH dafür kritisieren, dass er sich zu weit aus dem Fenster lehnt?

Müntefering: Als Politiker möchte ich darauf aufmerksam machen, dass das ein sehr komplexer Gesamtzusammenhang ist, in dem wir Politiker uns bewegen und bei dem 
leicht Fehler zum Schaden der Menschen, die konkret davon betroffen sind, passieren können. Besondere Komplexität kennzeichnet die Frage, ob man ein Gesetz auf nationaler oder europäischer Ebene machen soll. Das möchte ich mit folgendem Beispiel verdeutlichen: Die Regulierung des Arbeitmarktes ist ganz klar Sache der Mitgliedstaaten. Ferner muss heutzutage die staatliche Altersvorsorge dringend um die betriebliche Altersvorsorge ergänzt werden, was zunehmend gemacht wird. Nun gibt es aber eine Mobilität von hunderttausenden von Menschen, die sich in Europa bewegen. Diese Menschen fragen uns Politiker nach dem Schicksal ihrer Ansprüche aus der betriebliche Altersvorsorge, wenn sie den Arbeitgeber wechseln oder gar in einem anderen Mitgliedstaat der EU tätig werden. Ferner möchten sie wissen, ob es für diesen Themenkomplex eine einheitliche Regelung auf europäischer Ebene gibt, oder ob das in den Mitgliedstaaten unterschiedlich gehandhabt wird. Auch wenn wir die betriebliche Altersvorsorge in Deutschland insolvenzfest und mobil gestalten, bleibt immer noch die Frage, wie das die anderen Mitgliedstaaten regeln. Mit alledem ist auch zwangsläufig das Problem verbunden, was die nationalen Gerichte entscheiden und für was der EuGH eine Zuständigkeit hat. Welche Gesetze sind daher auf nationaler und europäischer Ebene zu beschließen, damit dieses Zusammenspiel funktionieren kann?

Europa ist kein Staat und wird das auf absehbare Zeit auch nicht sein. Wichtig ist daher das ehrliche Bemühen der Politik, eine solche Konstruktion, die einmalig ist, so zu organisieren, dass sie zum Nutzen der Menschen über klare Regeln verfügt, die die Menschen verstehen und die nicht gegen die Menschen ausgelegt werden können, weil entweder wir Politiker noch etwas vergessen hätten zu entscheiden, oder die Gerichte auf nationaler oder europäischer Ebene sich nicht klar sind darüber, wer denn eigentlich welche Entscheidungen zu treffen hat. Ganz selbstkritisch betrachtet glaube ich, dass es zu oft vorkommt, dass die Politiker in den einzelnen Mitgliedstaaten, Europa als Entschuldigung vorschieben, um von eigenen politischen Versäumnissen abzulenken.

Politik und Rechtsprechung müssen stärker lernen miteinander zu reden, aufeinander $\mathrm{zu}$ hören und gemeinsam zu überlegen, was man eigentlich tun kann, um dieses Europa $\mathrm{zu}$ einem Instrument zu machen, das regierbar ist, ohne eine Regierung zu haben und das für die Menschen transparent genug ist.

Möller: Sie haben gesagt, wir Politiker müssen lernen und ich sage jetzt ganz hart: die deutschen Politiker lernen nichts, weil sie Richter und Generalanwälte immer nur für sechs Jahre zum EuGH schicken. Stimmt es, Herr Skouris, dass wir Deutschen dadurch keinerlei Einfluss über unsere Richter auf die Rechtsprechung des EuGH bekommen?

Skouris: Dazu kann ich mich nicht äußern.

Möller: Das habe ich mir gedacht, weil das natürlich ein heikles Thema ist. Dann zurück in die Runde, was meinen Sie dazu, Frau Wendeling-Schröder? 
Wendeling-Schröder: Ich bin der Ansicht, dass zuviel Rotation schädlich ist. Die Arbeit beim EuGH ist so komplex, dass man eine ganze Weile braucht, bis man sich eingearbeitet hat. Daher ergibt sich schon allein aus der Natur der Sache, dass man nicht nur eine Periode da sein sollte. Wir haben im niedersächsischen Verfassungsgerichtshof versucht, dieses Spannungsfeld zwischen dem Bedürfnis nach Rotation auf der einen und dem nach Sachkenntnis auf der anderen Seite so zu lösen, dass man maximal zwei Legislaturperioden bleiben kann, was dann schon zwölf Jahre sind. Das ist eine gute Zeitspanne, die auch empfehlenswert für die deutsche Vertretung beim EuGH ist.

Müntefering: Heißt das, Sie unterstellen, dass die nationale Zugehörigkeit eines Richters Einfluss hat auf das, was er entscheidet?

Möller: Das will ich nicht sagen, aber wenn man länger da ist, hat man die Chance, zumindest Kammerpräsident zu werden. Vielleicht hätte ja dann auch mal eine Deutsche oder ein Deutscher die Möglichkeit, das höchste Amt am EuGH zu bekommen.

Müntefering: Sozusagen auch als Renommee für das Land.

Möller: Ja.

Müntefering: Nein nein. Das ist schon ein schwerwiegendes Problem, wenn ich darüber in der Politik diskutiere: Renommee für das Land oder für die Person. Soll ich ernsthaft in meinen Versammlungen behaupten, wenn eine Deutsche da säße, wäre es ein anderes Urteil? Das kann ich doch gar nicht machen. Das glaube ich auch gar nicht. Ich als Politiker muss da ganz vorsichtig sein und darf so etwas nicht unterstellen.

Möller: Aber würden Sie mir zustimmen, dass, je länger jemand am Hofe ist, er die dortigen Verfahren besser kennt und möglicherweise auch dasjenige besser mit einbringen kann, was er auf Seiten der deutschen Rechtsordnung sieht?

Skouris: Man kann davon ausgehen, dass auch bei einem Deutschen als Präsident des Gerichtshofs die Entscheidungen genauso ausfallen würden, wie sie uns heute vorliegen. Deswegen ist das sicherlich kein Kriterium. Ein anderer Punkt ist, dass es sicherlich eine Zeit dauert, bis man bei unserem System am Gerichtshof soweit eingearbeitet ist, dass man voll einsatzfähig wird. Daher ist aus funktionalen Gesichtspunkten ein Mandat von sechs Jahren möglicherweise zu kurz. Auch wird die Tätigkeit am EuGH für potentielle Kandidatinnen und Kandidaten aufgrund dieses kurzen Mandats nicht attraktiver, obwohl jedes Land ein Interesse daran haben sollte, die besten Leute da hinzuschicken, nicht weil man auf diese Weise Einfluss auf die Rechtsprechung nimmt, sondern weil die Rechtsprechung auf diese Weise besser wird. Vom Europäischen Par- 
lament wurde bereits einige Male vorgeschlagen, das Mandat auf zwölf Jahre zu verlängern, was aber der Rat nicht akzeptiert hat.

Schmidt: Meiner Meinung nach ändert eine Aufgabe oder Beinbehaltung des Rotationsprinzips nichts an den von Herrn Müntefering dargestellten Schwierigkeiten. Vielmehr kann sich die Politik nur selbst aus ihrem Dilemma befreien, indem klare, verständliche Gesetze gemacht werden, die klaren politischen Vorgaben folgen. Die Lösung besteht auch darin, in den mündlichen Verhandlungen beim EuGH die deutsche Sache so zu vertreten, dass die Botschaft, die man aus Deutschland an den EuGH senden möchte, dort auch ankommt.

Möller: Frau Koskelo, wir diskutieren ja hier viele Dinge so ein wenig aus deutscher Sicht. Deshalb sind wir froh, Sie als Gast aus Finnland in unserer Mitte zu haben. Ich habe ja gesagt, dass wir auch mal über den Tellerrand hinausschauen wollen. Wie wird die Richterberufung zum EuGH in Finnland gehandhabt?

Koskelo: Wir haben in diesem Bereich leider nicht viel Erfahrung, da wir erst den zweiten Richter beim EuGH haben. Aktuell wird in Finnland ein formaleres Verfahren mit mehr Konsultationen installiert, um die Benennungen vorzubereiten.

Aber gestatten Sie mir noch auf etwas zurückzukommen, was etwas mit dem zu tun hat, was Herr Müntefering gesagt hat. Es ist sehr wichtig für den EuGH, die Folgen seiner Entscheidungen genau abzusehen, zu verstehen und bedenken. Denn der EuGH kann nur feststellen, dass eine bestehende nationale Vorschrift nicht mit dem Gemeinschaftsrecht vereinbar ist. Er kann aber nichts an die Stelle der umstrittenen nationalen Vorschrift setzen. Oftmals haben Entscheidungen des EuGH zur Folge, dass Druck auf den europäischen Gesetzgeber erzeugt wird, da die in den Entscheidungen aufgeworfenen Probleme nicht vom nationalen Gesetzgeber gelöst werden können. Wenn die Mitgliedstaaten mit einer Entscheidung des EuGH nicht einverstanden sind, dann können sie die Gemeinschaftsregeln ändern, was in der Praxis sehr schwierig ist, weil die Gemeinschaft keine Zuständigkeit besitzt oder der Rechtsänderung unüberwindliche politische Schwierigkeiten im Wege stehen. Darin liegt das eigentliche Dilemma.

Möller: In der Bundesrepublik gibt es passend dazu das geflügelte Wort: „Über dem BVerfG wölbt sich der blaue Himmel, aber die Politiker können den Horizont verändern." Über dem EuGH wölbt sich auch der blaue Himmel, aber der Horizont kann nicht mehr verändert werden, das ist der entscheidende Unterschied. Das möchte ich am Schluss stehen lassen. Aber eine Frage habe ich dann doch noch Herr Skouris, wie ist denn das jetzt mit den 15 Richtern?

Skouris: Langfristig könnte sich vielleicht so eine Vorstellung durchsetzen, im Moment erscheint es mir jedoch nicht zweckmäßig. So kurz nach den beiden letzten Erwei- 
terungen mit insgesamt zwölf neuen Mitgliedstaaten ist es wichtig, dass pro Mitgliedstaat ein Richter am Gerichtshof tätig ist. Man darf nicht verkennen, dass es von enormer Bedeutung ist, die Kontakte zu den nationalen Gerichten auch von unserer Seite aufrecht zu erhalten. Das ist eine unserer ganz wichtigen Aufgaben beim Gerichtshof, nicht um die nationalen Gerichte zu beeinflussen, sondern um von diesen zu erfahren, was ihre Sorgen sind, damit wir in Zukunft besser die uns gestellten Aufgaben bewältigen. Aus diesem Grund kann ich mir eine Reduzierung auf 15 Richter im Moment nicht vorstellen. Im Übrigen würde das eine Vertragsänderung voraussetzen und ich glaube, dass mit dem künftigen Reformvertrag ohnehin noch genügend Probleme zu bewältigen sind, da muss man nicht ohne Not noch eins hinzufügen.

Möller: Herzlichen Dank. Wir sind damit am Ende der Diskussion. Ich bedanke mich bei den Mitdiskutanten dafür, dass Sie so offen gesprochen haben. Ich bitte nun den Vizekanzler und Arbeits- und Sozialminister der Bundesrepublik Deutschland, Herrn Müntefering, um sein Schlusswort, das den endgültigen Abschluss dieser Tagung bilden wird.

Müntefering: Vielen Dank Herr Möller. Ich bedanke mich beim EuGH, der einen guten Teil dazu beigetragen hat, dass Europa so gelungen ist, wie es gelungen ist. Wir neigen ja alle dazu, dieses Europa zu kritisieren, aber wenn man sich erinnert, dann ist das schon eine große historische Leistung, dass wir das hinbekommen haben und ich hoffe, dass es uns gelingt, den Weg weiterzugehen. Ich bedanke mich aber auch bei Ihnen allen, die gekommen sind und mit uns diskutiert haben.

Die Menschen fragen sich, ob die, die demokratisch legitimiert politische Macht haben, in der Lage sind, in einer sich verändernden globalisierten Welt in dieser europäisierten Region Politik zu gestalten und zwar so, dass die Menschen Vertrauen haben. Ich glaube, dass das eine riesige Herausforderung ist, in der wir uns da bewegen und dass es einer großen gemeinsamen Anstrengung bedarf, dass uns das gelingt. Die Menschen haben derzeit relativ wenig Vertrauen in die Politik, was sich auch bei Wahlen niederschlägt. Viele zweifeln an uns, damit müssen wir uns auseinandersetzen. Wir müssen aufpassen, dass nicht autoritäre oder schlimmere Systeme in der Welt schneller sind als wir und als demokratische Länder zeigen, dass wir in der Lage sind, die $\mathrm{Zu}-$ kunft zu gestalten, was gerade auch für Europa in einer besonderen Art und Weise gilt.

Ich glaube, dass die, die Recht sprechen in Europa, hohes Vertrauen genießen, was Ihnen eine hohe Verantwortung zuweist, der sie auch gerecht werden müssen. Die Menschen müssen sich sicher sein können, dass in Europa und den Mitgliedstaaten nachvollziehbar und verständlich entschieden und gehandelt wird

Zusammenfassende führt mich das alles zur Bitte, dass wir Politiker und Sie in den obersten und wichtigsten Gerichten so miteinander sprechen müssen, dass wir uns verstehen. Und wenn diese Tagung etwas zum gegenseitigen Verständnis von Justiz und Politik beigetragen hat, dann ist das erreicht worden, um was es mir ging. 
Danke Ihnen, kommen Sie gut nach Hause und behalten Sie uns in guter Erinnerung, es war gut gemeint und es soll auch was Gutes daraus werden. Herzliches Glück auf Ihnen miteinander!

Markus SCHÖN 
